\title{
Afetividade, Conflito Familiar e Problemas de Comportamento em Pré- Escolares de Famílias de Baixa Renda: Visão de Mães e Professoras
}

\author{
Familial Affectivity, Conflict And Behavioral \\ Problems In Preschool Children Of Low-Income Families: \\ Mothers And Teachers Perceptions \\ Afectividad, Conflicto Familiar Y Problemas \\ De Comportamiento En Preescolares De Familias \\ De Baja Renta: Visión De Madres Y Profesoras
}

Lia Mara Inês Albertoni

Rohenkohl

Universidade Regional

Integrada, Campus de

Erechim

Elisa Kern de Castro

Universidade do Vale

do Rio dos Sinos, São

Leopoldo
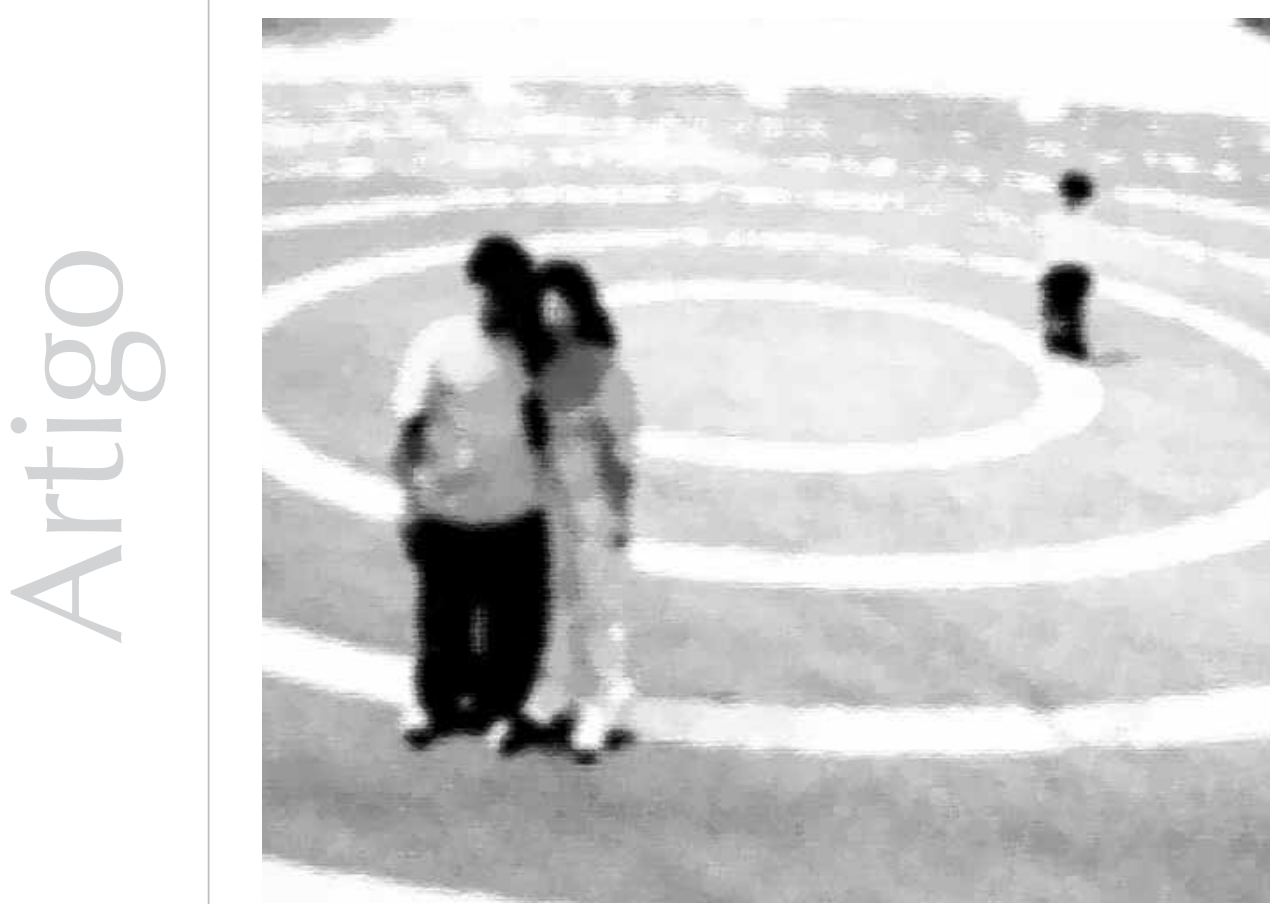
Resumo: A família é considerada um sistema social essencial na transmissão de crenças, ideias, conceitos e significados sociais, e influencia o comportamento das crianças. As relações afetivas estabelecidas na família constituem um dos fatores determinantes para o desenvolvimento emocional da criança. O objetivo deste estudo foi examinar o nível de afetividade e conflito em famílias de baixa renda e sua relação com os problemas de comportamento de crianças pré-escolares, a partir da visão das mães e das professoras. Participaram do estudo 59 mães de crianças em idade pré-escolar e suas respectivas professoras (10), que responderam a instrumentos sobre afetividade e conflito familiar e de problemas de comportamento infantil. Os dados revelaram a relação entre afetividade e conflito dos cônjuges e comportamento infantil. Quanto maior o grau de conflito entre os pais, maiores foram os problemas de comportamento das crianças, independentemente da configuração familiar.

Palavras-chave: Afeição. Conflito. Relação de objeto. Relações mãe-criança. Famílias de baixa renda. Distúrbios do comportamento. Crianças pré-escolares.

\begin{abstract}
The family is considered an essential social system in the transmission of beliefs, ideas and social meanings and has strong influence in children's behavior. The affectionate relationships established in the family are essential for the emotional development. The low-income families have peculiarities, but generally they try to approach the current social pattern. The objective of this study was to examine the level of affection and conflict in low-income families and their relation to behavior problems in preschool children from the point of view of mothers and teachers. The participants were 59 mothers of preschool children and their teachers, who responded to instruments on affection, family conflict and child behavior problems based on the mother's and teacher's point of view. The data highlighted the importance of the degree of affection and conflict between the father and mother, and its relationship with the children's development problems and that the greater the degree of conflict between the parents more behavior problems children develop, independently of the family structure.
\end{abstract}

Keywords: Family. Affection. Objects relations. Conflict. Behavior disorders. Mother child relations. Lowincome. Problems of behavior. Preschool age children.

Resumen: La familia es considerada un sistema social esencial en la transmisión de creencias, ideas, conceptos y significados sociales, e influencia el comportamiento de los niños. Las relaciones afectuosas establecidas en la familia constituyen uno de los factores determinantes para el desarrollo emocional del niño. El objetivo de este estudio ha sido el de examinar el nivel de afectividad y conflictos en familias de baja renta y su relación con los problemas de comportamiento de niños preescolares, desde la perspectiva de las madres y de las profesoras. Han participado del estudio 59 madres de niños en edad preescolar y sus respectivas profesoras (10), que han respondido instrumentos acerca de la afectividad y conflicto familiar $y$ de problemas de comportamiento infantil. Cuanto mayor es el grado de conflicto entre los padres, mayores han sido los problemas de comportamiento de los niños, independientemente de la configuración familiar. Palabras clave: Afeccion. Conflicto. Relaciones de objeto. Transtornos de la conducta. Relaciones madreniño. Familias de baja renta. Problemas de comportamiento. Niños preescolares.

A família é o principal meio de socialização por introduzir crenças, cultura e modos de pensar, construindo sujeitos e cidadãos. É nela que ocorrem as primeiras relações e identificações com figuras importantes e, portanto, onde se produzem os primeiros comportamentos da criança (Amazonas, Damasceno, Terto, \& Silva, 2003; Gomide, 2004; Kreppner, 2000). As relações afetivas estabelecidas na família constituem um determinante para o seu desenvolvimento emocional (Mondin, 2005), pois é a partir dela que as crianças aprendem as diferentes formas de ver o mundo e constroem as suas relações sociais.
As experiências familiares oportunizam a formação de repertório de comportamentos, de ações e resoluções frente aos problemas (Dessen \& Polônia, 2007). É no ambiente familiar também que a criança aprende a lidar com os conflitos, a controlar suas emoções, a demonstrar os diferentes sentimentos que permeiam as relações e a lidar com a vida e com suas adversidades.

Para Teodoro, Cardoso e Freitas (2009), a família é essencial para a formação e a socialização da criança, além de proporcionar uma estrutura de apoio para lidar com os problemas sociais, escolares e financeiros. 
A qualidade dos laços afetivos formados entre pais e filhos pode ser considerada

preditora de um desenvolvimento saudável e, em consequência, de interações e de padrões de ajustamento positivos em todos os ambientes em que participam (Dessen e Polônia, 2007).
Nesse sentido, para esses autores, é de grande importância identificar os aspectos do funcionamento do sistema familiar que podem estar relacionados tanto ao bom funcionamento emocional e cognitivo como às psicopatologias. Os pais proporcionam, ou deveriam proporcionar, todo o suporte necessário para que o desenvolvimento saudável ocorra, além de funcionarem como mediadores das questões sociais (Baptista \& Oliveira, 2004; Rocha \& Brandão, 2001).

A qualidade dos laços afetivos formados entre pais e filhos pode ser considerada preditora de um desenvolvimento saudável e, em consequência, de interações e de padrões de ajustamento positivos em todos os ambientes em que participam (Dessen e Polônia, 2007). No entanto, esses mesmos laços afetivos podem dificultar o desenvolvimento da criança e provocar problemas no seu ajustamento social.

Benetti (2006) afirma que os conflitos entre o casal podem se apresentar através de discussões e brigas ou velados, manifestados por boicotes e indiferença, dentre outros. Considerando-se que toda relação envolve certo nível de conflito, é importante entender que aspectos do desenvolvimento infantil são afetados por ele (Cummings, 1995). Nesse sentido, alguns estudos brasileiros (Teodoro, 2006; Teodoro \& Kappler, 2003) iniciaram essa discussão e relacionaram a importância que têm o afeto e o conflito no sistema familiar na qualidade de vida das crianças.

A afetividade refere-se ao conjunto de emoções positivas que existem no relacionamento interpessoal (Baptista, Teodoro, Cunha, Santana, \& Carneiro, 2009). Ela implica uma relação de carinho e cuidado que se tem com alguém. Os laços afetivos proporcionam apoio psicológico e social na família, auxiliando no enfrentamento das dificuldades do cotidiano; o conflito, em contrapartida, é caracterizado por sentimentos negativos que podem gerar estresse e agressividade no sistema familiar, sendo que, por conflito, entende-se a luta interna individual entre as necessidades e impulsos ou as exigências internas e externas opostas ou incompatíveis.

A relação entre pais, mães e filhos no início da vida está associada a uma série de comportamentos por meio dos quais o indivíduo inicia e mantém relações afetivas estáveis (Zamberlan, 2002). A relação entre os pais tem sido apontada por Braz, Dessen e Silva (2005) como fator importante para a qualidade de vida das famílias, influenciando nos cuidados com os filhos e nas relações entre pais e filhos. Ainda, o apoio dos pais para com as mães possibilita um desenvolvimento saudável aos filhos. Por outro lado, alguns estudos (Keown \& Woodward, 2002; Ferriolli, Marturano, \& Puntel, 2007) têm demonstrado que, entre as possíveis causas de problemas emocionais e de comportamento em crianças pré-escolares, estão fatores familiares, separações, doenças psiquiátricas em um dos cônjuges, tamanho e agressões dentro da família, dificuldade dos pais em estabelecer limites, acontecimentos importantes na vida da criança, negligência, comunicação e sintonia entre pais e filhos, entre outros. Patterson, Reid e Dishion (1992) acrescentam ajustamento do casal, conflitos entre os pais, problemas financeiros, desvantagem social dos pais, vizinhança e relacionamento entre pais e filhos como possíveis variáveis que podem facilitar o surgimento ou a manutenção de problemas de comportamento em pré-escolares.

O desenvolvimento infantil é marcado por mudanças de comportamentos considerados importantes por permitirem à criança a aprendizagem de novas habilidades, e podem ser caracterizados como desejáveis ou indesejáveis (Bolsoni-Silva, Marturano, \& Manfrinato, 2005). Desejadas ou adequadas são as mudanças que ocorrem como 
consequência da interação do organismo com o ambiente, permitindo à criança o contato com situações importantes de aprendizagem que promovem o desenvolvimento. Os comportamentos indesejáveis referem-se aos problemas de comportamento decorrentes do excesso ou da falta de situações que facilitariam ou ampliariam as possibilidades de aprendizagem e, em consequência, de desenvolvimento. Estes são manifestados através de comportamentos internalizantes ou externalizantes (Achenbach, 1991; BolsoniSilva et al., 2005). Como comportamentos internalizantes, destacam-se retraimento e ansiedade, preocupação exagerada, tristeza, insegurança, timidez, medos, manifestações psicossomáticas e recusa escolar, dentre outros. Os comportamentos externalizantes, em contrapartida, são marcados pela impulsividade, explosividade, agressividade, agitação, características desafiantes e antissociais como mentiras, furtos, faltar à aula, desrespeito a limites, brigas e hostilidade nos relacionamentos (Achenbach, 1991). Esses são comportamentos que dificultam a interação da criança com o ambiente, geram conflitos e frequentemente ocasionam o rompimento nas interações. Geralmente, no contexto escolar, o comportamento externalizante é mais facilmente identificado, provavelmente por ter maior visibilidade e interferir na dinâmica da sala de aula. Dessa forma, a escola passa a ser um espaço importante de prevenção, tendo o professor o papel fundamental de identificar os alunos com dificuldades e, assim, de possibilitar-lhes o desenvolvimento de habilidades sociais e relacionais (Ferriolli, Marturano, \& Puntel, 2007).

Estudos internacionais têm apontado que a qualidade das relações familiares pode ser um fator de proteção para o surgimento de problemas psicológicos na criança (Althoff, 2008; Feinberg, Button, Neiderhiser, Reis, \& Hetherington, 2007). Por outro lado, também tem sido verificada a relação entre problemas de comportamento nas crianças e estresse e doença mental nos pais (Pekrun, 2001; Rollett \& Werneck, 2002).

Alguns estudos brasileiros recentes têm investigado a questão dos problemas de comportamento e situação familiar. BolsoniSilva e Marturano (2010) avaliaram o relacionamento conjugal de 48 casais e sua relação com problemas de comportamento em pré-escolares. Os achados confirmaram em parte a ideia de que a ocorrência de conflitos familiares pode ser uma variável que favoreça o surgimento de problemas de comportamento em pré-escolares, uma vez que os pais/mães das crianças com problemas de comportamento manifestaram dificuldades comportamentais que dificultavam a relação entre pais e filhos. No entanto, pesquisas que avaliam a concordância de pais e professoras sobre comportamento de crianças préescolares são escassas no contexto brasileiro. Borsa e Nunes (2008) constataram que pais e mães tiveram nível de concordância de baixo a moderado sobre o comportamento infantil medido através do $\mathrm{CBCL}$, o que mostra que mesmo pessoas com contato próximo à criança podem proporcionar informações discordantes sobre o seu comportamento. A pesquisa realizada por Bolsoni-Silva, Marturano, Pereira e Manfrinato (2006) com mães e professoras teve como objetivo de comparar as avaliações sobre habilidades sociais e problemas de comportamento em crianças consideradas problemáticas pelas professoras. Os autores observaram que as avaliações sobre habilidades sociais em crianças sem problemas de comportamento foram semelhantes entre mães e professoras; entretanto, nas crianças que manifestavam problemas, as avaliações das habilidades sociais e dos problemas eram diferentes, pois as mães os percebiam com mais habilidades e menos problemas. Esses resultados mostram a importância de considerar a opinião de pessoas que convivem em diferentes contextos com as crianças para melhor 
compreender os seus possíveis problemas ou dificuldades.

Tendo em vista a importância de investigar aspectos relacionados aos problemas de comportamento de pré-escolares e características familiares, os objetivos deste trabalho foram: 1) examinar o nível de afetividade e conflito em famílias de baixa renda e correlacioná-los com os problemas de comportamento em crianças pré-escolares, a partir da visão das mães e das professoras e 2) comparar a percepção de mães e de professoras em relação aos problemas de comportamento das crianças pré-escolares, identificando, também, possíveis diferenças nessa percepção conforme o sexo da criança.

\section{Método}

\section{Participantes}

Participaram desta pesquisa 59 mães com filhos em idade pré-escolar, residentes em uma cidade do interior de um Estado brasileiro, de nível socioeconômico baixo, com crianças que tinham entre dois e cinco anos e que frequentavam uma escola municipal da periferia; participaram também as professoras dessas crianças (dez). A amostra foi escolhida por conveniência, ${ }^{1}$ e todos os participantes convidados aceitaram participar da pesquisa.

Do total de mães, a grande maioria possuía ensino fundamental incompleto $(74,6 \%)$. Com relação à profissão, $37,3 \%$ eram donas de casa, 42,4\% eram funcionárias da iniciativa privada e exerciam atividades como higienistas, cozinheiras, domésticas e serviços gerais, dentre outros, e 18,5\% executavam trabalhos informais como coletoras de lixo, carrinheiras, camelôs ou faxineiras. Dos pais, a grande maioria também possuía ensino fundamental incompleto (76,8\%). Os trabalhos mais frequentes foram trabalho informal (54,9\%) (catador de erva mate, pintor, encanador, catador de lixo e camelô, dentre outras) e trabalho na iniciativa privada $(37,7 \%)$ como mecânico, vigilante, padeiro, construção civil, gari ou chapeador. Cabe destacar o alto índice de união estável e casamentos (juntos correspondiam a 78,7\% das famílias) sendo que destes, $52,5 \%$ eram famílias nucleares, 25,2\% reconstituídas e $9,9 \%$ uniparentais. A média de filhos por mãe foi de 1,97, enquanto a média de idade das mães foi de 31,05 anos. As crianças possuíam idade média de 4,16 anos, sendo $29(49,2 \%)$ do sexo feminino e $30(50,8 \%)$ do sexo masculino. O número de habitantes por domicílio era em média 4,27, o que demonstra convivência com a família extensa (avós, tios).

Para avaliar o nível socioeconômico, foi utilizada a escala proposta por Hollingshead (1975) já empregada em outros estudos brasileiros (Ribas, Seidl de Moura, Gomes, Soares, \& Bornstein, 2003; Prado, 2005) que o calcula a partir do nível educacional, do prestígio ocupacional e do tipo de emprego. Observou-se que a amostra era constituída por famílias de nível socioeconômico baixo.

\section{Instrumentos}

Fichas de matrícula da escola: usadas para avaliar as informações relativas aos dados biossociodemográficos das famílias, tais como tipo de família, número de filhos, condições sócio-econômicas e especificidades do desenvolvimento do aluno (condições de saúde, desenvolvimento, relacionamento).

Familiograma (Teodoro, 2006): visa a investigar o relacionamento entre as díades (filho, pai e mãe) através de adjetivos que representam duas dimensões do sistema familiar: a afetividade e o conflito. A afetividade definese por uma série de emoções positivas existentes no relacionamento interpessoal, tais como amável, afetivo, amoroso, carinhoso, alegre, feliz, atencioso, animado, agradável. 
O conflito é caracterizado por emoções negativas que podem ser fonte geradora de estresse e agressividade dentro das famílias, como distante, nervoso, agressivo, estressante e tenso. A lista de adjetivos para medir afetividade e conflito contém 14 vocábulos. $\mathrm{O}$ instrumento demonstrou ter boa consistência interna (alpha de Cronbach variando de 0.87 a 0,97 ), e vem sendo utilizado em famílias com características distintas. O familiograma pode ser utilizado tanto nas diversas configurações familiares como também em relacionamentos em famílias clínicas e não clínicas. Neste estudo, o instrumento foi aplicado para avaliar a relação das díades mãe-filho e mãe-pai, e obteve índices alpha de 0,95 para afetividade e 0,87 para o fator conflito para a díade mãe-filho. Para a díade mãe-pai, o alpha foi de 0,97 para o fator afetividade e de 0,91 para o fator conflito. A classificação do tipo de família, de acordo com os constructos afetividade de conflito no familiograma, é: tipo I - as famílias com alta afetividade e baixo conflito; tipo II - famílias com alta afetividade e alto conflito; tipo III - famílias com baixa a afetividade e baixo conflito e tipo IV - famílias com baixa afetividade e alto conflito.

Child Behavior Checklist (CBCL) - 1,1/2 - 5 anos - e Teacher's Report Form (TRF): foram utilizadas duas versões do checklists de problemas comportamentais de Achenbach (1991), voltadas para pais e professores, na versão brasileira realizada por Bordin, Mari e Caiero (1995). O Child Behavior Checklist (CBCL 1.1/2-5) foi o instrumento preenchido pelas mães, enquanto o Teacher's Report Form (TRF), pelas professoras. Esses instrumentos foram desenvolvidos com uma lista de 100 afirmações sobre o comportamento da criança, distribuídas em duas colunas e seguidas por uma escala preenchida pelo entrevistado. A escala correspondia à quantidade de vezes que cada afirmação do questionário se aplicava àquela criança que estava sendo analisada. Os valores da escala eram 0 (nunca), 1 (às vezes) e 2 (sempre). Essas listas de afirmações foram agrupadas em oito síndromes, através de uma análise fatorial (Achenbach, 1991): isolamento social, queixas somáticas, ansiedade-depressão, problemas sociais, problemas do pensamento, problema de atenção, comportamento agressivo, problemas de sono e outros problemas. Tais síndromes receberam uma análise fatorial de segunda ordem que resultou em agrupamentos que foram designados de: 1) distúrbio internalizante (DI) - comportamentos considerados problemáticos, mas que não interferem no ambiente, restringindo-se ao âmbito privado da criança e 2) distúrbio externalizante (DE) - refere-se a comportamentos problemáticos que exercem interferência diretamente no ambiente. De acordo com a versão brasileira (Bordin et al., 1995), crianças cujos escores nos $\mathrm{CBCL}$, preenchidos pelos pais, foram iguais ou acima de 60 foram consideradas pertencentes à faixa clínica.

\section{Delineamento e procedimentos de pesquisa e éticos}

Este é um estudo transversal e comparativo porque compara, em um momento dado, a visão das mães e das professoras sobre o comportamento das crianças. A coleta de dados realizou-se nas dependências da escola, onde todos os bimestres as mães são convidadas a participar de uma reunião com as professoras para que possam conversar sobre os filhos. Os instrumentos foram aplicados individualmente e preenchidos pela entrevistadora a partir das respostas das mães, em função da baixa escolaridade e da dificuldade de leitura e de compreensão de texto que muitas delas apresentavam. Com as professoras, o instrumento foi aplicado alguns dias após a aplicação nas mães, individualmente, no ambiente escolar.

O trabalho foi aprovado pelo Comitê de Ética em Pesquisa (instituição omitida), projeto 
no 08/026 de 06/05/2008, conforme a Resolução no 196/96 do Conselho Nacional de Saúde. Os participantes receberam explicações sobre todos os procedimentos da pesquisa, assim como a respeito do sigilo sobre a sua identidade. Também foram informados de que não haveria riscos ou danos para eles. Ao aceitarem participar do estudo, assinaram o Termo de Consentimento Livre e Esclarecido antes da aplicação dos instrumentos.

\section{Análise dos dados}

As informações foram tabuladas e analisadas em um banco de dados do programa SPSS 17.0. Inicialmente, realizou-se uma análise descritiva (médias, DP, porcentagens) dos resultados em geral e sua distribuição por quartis. Para analisar as associações entre variáveis, realizaram-se correlações de Pearson. Foi feita a comparação de médias (Teste t pareado) dos resultados das mães e professoras e dos resultados conforme o sexo da criança (Teste t independente).

\section{Resultados}

Inicialmente, realizaram-se análises descritivas dos resultados do $\mathrm{CBCL}$, do TRF e do familiograma. A Tabela 1 apresenta as médias, as medianas, os quartis e os valores mínimo e máximo dos resultados desses instrumentos.

Tabela 1. Resultados descritivos do familiograma (FG) $(n=58)$, do Child Behavior Checklist $(\mathrm{CBCL})(\mathrm{n}=59)$ e do Teacher's Reports Form (TRF) $(n=59)$

\begin{tabular}{lllllll}
\hline Instrumento & Fator & Média(DP) & Mediana & 10 Quartil & 3o Quartil & Min/Max \\
\hline CBCL & CBCL total & $49.92(18.24)$ & 48.00 & 38.00 & 48.00 & $16-92$ \\
& CBCL internalizante & $15.20(7.78)$ & 14.00 & 9.00 & 14.00 & $2-32$ \\
\multirow{2}{*}{ TRF } & CBCL externalizante & $17.93(7.55)$ & 17.00 & 13.00 & 17.00 & $2-37$ \\
& TRF total & $43.23(25.34)$ & 41.00 & 24.00 & 56.00 & $0-106$ \\
& TRF internalizente & $13.49(9.85)$ & 12.00 & 5.50 & 18.50 & $0-42$ \\
FG Afetividade & TRF externalizante & $15.52(12.78)$ & 13.00 & 4.50 & 25.00 & $0-41$ \\
& Mãe-pai & $47.36(5.28)$ & 46.00 & 44.00 & 52.50 & $33-55$ \\
FG & Mãe-filho & $41.25(9.37)$ & 43.50 & 37.00 & 47.00 & $18-55$ \\
Conflito & & & & & & $11-41$ \\
& Mãe-pai & $20.87(8.57)$ & 19.00 & 13.25 & 26.00 & $11-43$ \\
\hline
\end{tabular}

Foram realizadas análises relativas às porcentagens dos níveis normais, limítrofes e clínicos do CBCL e do TRF e aos níveis de conflito e de afetividade nas famílias de acordo com o familiograma. No CBCL e no TRF, o ponto de corte para que uma criança fosse considerada limítrofe dizia respeito ao percentil 75, e, para o grupo clínico, foi utilizado o percentil 90. Esse critério foi o mesmo usado no estudo de Silvares e Melo (2003). Para o familiograma, utilizou-se a mediana para fazer os pontos de corte para alta e baixa afetividade e conflito, conforme estudo de Baptista et al. (2009). Os resultados apontam um percentual elevado $(72,1 \%)$ de baixa afetividade e de alto conflito $(45,9 \%)$ na díade mãe e pai. Os resultados do CBCL e do TRF total mostraram que a maior parte das crianças se encontrava nos padrões considerados normais, sendo que $14,8 \%$ dos casos foram considerados limítrofes e 11,5\%, clínicos. 
Ao avaliar os tipos de família encontrados a partir do familiograma, observou-se que a maioria das famílias era do tipo I, com alta afetividade e baixo conflito (52,5\%); 31,1\% das famílias era do tipo II, que apresentava alta afetividade e alto conflito; $3,3 \%$ era do tipo III, que demonstrava baixa afetividade e baixo conflito, e 13,1\% era do tipo IV, com baixa afetividade e alto conflito. Optouse por chamar as famílias do tipo I de afetivas, as do tipo II, intensas, as do tipo III, inconsistentes, e as do tipo IV, conflitivas.

Utilizou-se o Teste t pareado para verificar possíveis diferenças nas percepções de mães e professoras com respeito aos comportamentos infantis (medidos pelo $\mathrm{CBCL}$ ) e para medir possíveis discrepâncias entre as díades mãe-filho e mãe-pai e/ou companheiro na afetividade e conflito familiar (medidos através do familiograma). Observou-se que as mães e pais perceberam as crianças como mais problemáticas do que as professoras na escala de problemas total $(t=2,045, p<0,05)$ e nas subescalas queixas somáticas ( $t=3,132, p<0,001)$ e ansiedade e depressão $(t=4,202$, $p<0,001)$. Em relação ao familiograma, os resultados mostraram que a díade pai-mãe demonstrou maior conflito ( $t=3,562, p<0,001)$ que a díade mãe-filho, e a díade mãe-filho demonstrou maiores níveis de afetividade de maneira significativa $(t=-5,075, p<0,001)$, conforme mostra a Tabela 2.

Tabela 2. Comparação de médias (Teste t pareado) dos resultados do CBCL respondido pela mãe $(n=59)$, do TRF respondido pela professora $(n=59)$ e familiograma $(n=58)$

\begin{tabular}{lccc}
\hline & Média (DP) & Teste t pareado & $\mathrm{p}<$ \\
\hline CBCL/TRF total & & \\
Mãe & $49,92(18,23)$ & & \\
Professora & $43,23(25,34)$ & 2,045 & 0,05
\end{tabular}

$\mathrm{CBCL} / \mathrm{TRF}$ internalizante

Mãe

Professora

n.s.

$\mathrm{CBCL/TRF}$ externalizante

Mãe

Professora

n.s.

Isolamento social

Mãe

Professora

n.s.

Queixas somáticas

Mãe

Professora

Ansiedade e depressão

Mãe

Professora

Reações emocionais

Mãe

Professora

n.s.

Problemas de sono 
Mãe

Professora

Comportamento agressivo

Mãe

Professora

Outros problemas

Mãe

Professora

Conflito

Mãe-pai

Mãe-filho(a)

Afetividade

Mãe-pai

Mãe-filho
$3,30(2,32)$

$3,21(3,20)$

$14,64(6,76)$

$12,31(10,17)$

1,645

n.s.

$10,77(8,06)$

2,663

n.s.

$20,87(8,57$

$16,92(6,77)$

3,582

0,001

$41,25(9,36)$

$47,35(5,32)$
$-5,075$

0,001

Ao realizar a comparação entre os resultados do CBCL e do TRF em relação ao conflito e à afetividade das díades mãe e pai e mãe e filho (amostra separada pela mediana), observou-se que, nas famílias com alto conflito mãe-pai, as crianças apresentaram mais problemas emocionais/ comportamentais no total $(\mathrm{t}=-4,202, \mathrm{p}<0,001)$ que as famílias com baixo conflito. Da mesma forma, nas famílias com alto conflito mãe-pai e baixa afetividade mãe-filho, as crianças tiveram maiores escores em problemas de internalização $(t=-3,761, p<0,001 ; t=2,056, p<0,05$, respectivamente). Por outro lado, nas famílias com alto conflito mãe-pai e alto conflito mãefilho, as crianças manifestaram mais problemas externalizantes $(t=-3,014, p<0,01 ; t=-2,097$, $p<0,05)$. Quanto à existência de possíveis diferenças de gênero em afetividade e conflito e em problemas de comportamento entre as díades mãe-filha e mãe-filho, medidos através do Teste $t$ independente, os resultados mostraram que as meninas apresentavam maior índice de queixas somáticas $(\mathrm{t}=-2,744 ; \mathrm{p}<0,01)$ que os meninos.

A análise de correlação de Pearson mostrou que os níveis de conflito das díades mãe-pai se correlacionaram de maneira significativa com as dimensões internalizante $(r=0,338, p<$ $0,01)$ e externalizante $(r=0,455 ; p<0,001)$ e com a pontuação total do CBCL $(r=0,457 ; p<$ 0,001); ainda, os níveis de conflito das díades mãe-filho tiveram correlação com as dimensões internalizante $(r=0,266, p<0,05)$, externalizante $(r=0,286, p<0,05)$ e total $(r=0,341, p<0,01)$. Com relação à afetividade mãe-pai, obtiveram-se correlações negativas e significativas com o CBCL total $(r=-0,294, p<0,05)$ e com a dimensão externalizante $(r=-0,316, p<0,05)$, enquanto, nas díades mãe-filho, a única correlação significativa e negativa foi com a dimensão internalizante $(r=-0,330, p<0,01)$. De forma geral, quanto maior a afetividade e menor o conflito nessas famílias, seja nas díades mãe-filho ou mãe-pai/companheiro, menores os problemas de 
comportamento das crianças. Por último, observa-se correlação significativa entre afetividade mãe-pai/companheiro $(r=$ -0,782, $p<0,001)$ e mãe-filho $(r=0,281$, $p<0,05)$ e conflito mãe-pai/companheiro e mãe-filho $(r=0,344, p<0,001)$.

\section{Discussão}

O objetivo principal desta pesquisa foi examinar o nível de afetividade e conflito em famílias de baixa renda e sua relação com os problemas de comportamento de crianças pré-escolares a partir da visão das mães e das professoras. Dessa forma, buscou-se verificar se existiam diferenças, na percepção de mães e de professoras, em relação aos problemas de comportamento das crianças pré-escolares, identificando-se, também, possíveis discrepâncias nessa percepção conforme o gênero da criança.

Os resultados relativos aos problemas de comportamento mostraram que as mães perceberam mais esses problemas que as professoras. Esse fato pode ocorrer porque a convivência da mãe com os filhos se dá dentro de um grupo menor que na escola, o que permite a elas perceber nuances do comportamento das crianças de forma mais perspicaz que as professoras. Por outro lado, existem outros fatores que devem ser levados em consideração, como o tempo de permanência da criança na escola e em casa e a qualidade das interações que se dão nesses ambientes. No entanto, há indícios de que as crianças diferenciam o ambiente escolar do ambiente de casa, podendo ser mais contidas e cooperativas na escola (D'Avila-Bacarji, 2005; Pacheco \& Sisto, 2005).

Através dos resultados desta pesquisa, pôde-se constatar que não houve diferença de sexo das crianças quanto à percepção das mães sobre possíveis problemas de comportamento, bem como no nível de afetividade e conflito de suas famílias.
Entretanto, percebeu-se maior relato de manifestação de queixas somáticas nas mães de meninas, o que coincide com a literatura existente (Moura, Marinho-Casanova, Meurer, \& Campana, 2008). Pode existir também a expectativa, por parte dos adultos, de que as meninas sejam quietas e comportadas, o que pode levar a uma internalização de suas dificuldades, também considerada um problema de comportamento. Os problemas de internalização, segundo LaFreniere e Dumas (1996), podem dificultar seu desenvolvimento social e reduzir as suas oportunidades de interação com os pares. Nesse sentido, é fundamental considerar os contrastes entre o ambiente escolar e familiar e as diferenças de condições em que a mãe e as professoras fazem sua avaliação.

Os dados revelaram também a importância do grau de afetividade e conflito entre os cônjuges e sua relação com os problemas de comportamento infantil, independentemente da configuração familiar. A família continua tendo papel fundamental no desenvolvimento de seus membros, e ambientes conflitivos desfavorecem o desenvolvimento da criança (Althoff, 2008; Ferriolli et al., 2007; Keown \& Woodward, 2002). Investir em programas que possibilitem instrumentalizar os pais para lidarem de forma mais adequada com a família e com os filhos poderia reduzir o grau de conflito e, em consequência, auxiliar no desenvolvimento saudável das crianças.

Diferentes autores analisaram a importância do suporte dos pais em relação ao desenvolvimento dos filhos, e afirmam que o desempenho em relação ao seu papel e ajuste social está relacionado tanto com as interações entre pais e filhos (Aunola, Stattin, \& Nurmi, 2000), com o desempenho escolar (Chen, Liu, \& Li, 2000; Connell \& Prinz, 2002), como também com a sua própria Saúde Mental (Pekrun, 2001; Rollett \& Werneck, 2002). A responsabilidade dos pais está associada à capacidade da criança de se 
ajustar ao ambiente em que vive como ao seu desempenho social e intelectual (Alvarenga \& Piccinini, 2001).

O nível socioeconômico e o contexto têm implicações na formação dessas famílias. Quando se pensa em famílias de baixa renda, deve-se levar em consideração a sua condição de vida, pois esta se reflete nos padrões de interação que estabelecem seus membros. Nesse sentido, é importante examinar as características dessas famílias no nosso contexto social e cultural, pois os resultados de estudos internacionais não necessariamente são semelhantes aos daqui. As crenças e valores dos pais constituem o ponto de contato com a cultura social e pessoal, e influenciam as suas práticas educativas com os filhos (Szelbracikowski \& Dessen, 2007). A forma como os pais educam os filhos vai se refletir nas suas relações posteriores. É fundamental para melhor compreensão dessa complexidade que se busquem subsídios em outras áreas, como educação, saúde, Sociologia e Antropologia.

Dentre as possíveis limitações deste estudo, uma delas se refere à fonte de dados dos participantes, que foram coletados com base na perspectiva materna e na da professora, o que faz pensar que a realidade vista sob outras perspectivas (a do pai e a das crianças) possa ser diferente. No entanto, obter informações de crianças pequenas é uma tarefa complexa, tendo em vista o pouco desenvolvimento cognitivo para responder a tais questões. Grande parte dos estudos realizados com famílias com crianças pequenas também obteve informações apenas de adultos e, na maior parte, das mães, como se verifica em Moura et al., (2008) e Pesce (2009). Outra limitação é que não foi possível fazer comparações sobre os problemas de comportamento infantis a partir dos quatro tipos de famílias descritas no familiograma, já que um reduzido número de famílias se encaixou nos tipos III e IV.

\section{Considerações Finais}

Ao término deste estudo, surgem novas ideias de investigação e de continuidade dentro dessa temática, com trabalhos que investiguem como se dá a percepção das crianças em relação aos pais e ao funcionamento familiar e como percebem a família extensa. A participação da comunidade e da escola na educação e na criação das crianças de famílias de baixa renda pode ser outra vertente de investigação útil para entender a relação família-escola. 


\section{Lia Mara Inês Albertoni Rohenkohl}

Mestrado em Psicologia pela Universidade do Vale do Rio dos Sinos. Professor titular da Universidade Regional Integrada do Alto Uruguai e das Missões Erechim e Psicólogo Clínico do Consultório Particular, Rio Grande do Sul - RS - Brasil.

E-mail: rohenkohl@uri.edu.br

\section{Elisa Kern de Castro}

Mestrado em Psicologia pela Universidade Federal do Rio Grande do Sul. Professora adjunta da Universidade do Rio dos Sinos, Rio Grande do Sul - RS - Brasil.

E-mail: elisa.kerndecastro@gmail.com

\section{Endereço para envio de correspondência:}

Centro de Ciências da Saúde - Universidade do Vale do Rio dos Sinos

Av. Unisinos, 950, bairro Cristo Rei. São Leopoldo - Rio Grande do Sul - RS - Brasil. CEP: 93022-000

Recebido 8/9/2010, 1a Reformulação 28/11/2011, Aprovado 10/1/2012. 


\section{Referências}

Achenbach, T. M. (1991). Manual for the Child Behavior Checklist/4-18 and 1991 profile. Burlington, VT: University of Vermont, Department of Psychiatry.

Achenbach, T. M. (1991). Manual for the Teacher's Report Form and 1991 profile. Burlington, VT: University of Vermont, Department of Psychiatry.

Alvarenga, P., \& Piccinini, C. (2001). Práticas educativas maternas e problemas de comportamento em pré-escolares. Psicologia: Reflexão e Crítica, 14(3), 449-460.

Amazonas, M. C. L. A., Damasceno, P. R., Terto, L. M. S., \& Silva, R. R. (2003). Arranjos familiares de crianças de camadas populares. Psicologia em Estudo, 8(Especial), 11-20.
Althoff, R. R. (2008). Diagnoses, neuropsychological functioning, and parental depression affect the expression of internalizing and externalizing disorders in children. Journal of the American Academy of Child \& Adolescent Psychiatry, 4, 358-358.

Aunola, K., Stattin, H., \& Nurmi, J. (2000). Parenting styles and adolescents' achievement strategies. Journal of Adolescence, 23, 205-222.

Baptista, M. N., \& Oliveira, A. A. (2004). Sintomatologia de depressão e suporte familiar em adolescentes: um estudo de correlação. Revista Brasileira de Crescimento e Desenvolvimento Humano, 14, 58-67.

Baptista, M. N., Teodoro, M. L. M., Cunha, R. V., Santana, P. R., \& Carneiro, A. M. (2009). Evidência de validade entre o inventário de percepção de suporte familiar - IPSF e familiograma - FG. Psicologia: Reflexão e Crítica, 23(2), 466-473. 
Benetti, S. P. C. (2006). Conflito conjugal: impacto no desenvolvimento psicológico da criança e do adolescente. Psicologia: Reflexão e Crítica, 19(2), 261-268.

Bolsoni-Silva, A. T., Marturano, E. M., \& Manfrinato, J. W. de S. (2005). Mães avaliam comportamentos socialmente "desejados" e "indesejados" de pré-escolares. Psicologia em Estudo, 10(2), 245-252.

Bolsoni-Silva, A. T., Marturano, E. M., Pereira, V. A., \& Manfrinato, J. W. S. (2006). Habilidades sociais e problemas de comportamento de pré-escolares: comparando avaliações de mães e de professoras. Psicologia: Reflexão e Crítica, 19(3), 460-469.

Bolsoni-Silva, A. T., \& Marturano, E. M. (2010, jan./mar.). Relacionamento conjugal, problemas de comportamento e habilidades sociais em pré-escolares. Psic.: Teor. e Pesq. 26(1), 67-75.

Bordin, I. A. S., Mari, J. J., \& Caeiro, M. F. (1995). Validação da versão brasileira do "Child Behavior Checklist" - CBCL (Inventário de Comportamentos da Infância e Adolescência): dados preliminares. ABP-APAL, 17(2), 55-66.

Borsa, J. C., \& Nunes, M. L. T. (2008). Concordância parental ao CBCL. Paidéia, 18(40), 317-330.

Braz, M. P., Dessen, M. A., \& Silva, N. L. P. (2005). Relações conjugais e parentais: uma comparação entre famílias de classes sociais baixa e média. Psicologia: Reflexão e Crítica, 18(2), 151-161.

Chen, X., Liu, M., \& Li, D. (2000). Parental warmth, control, and indulgence and their relations to adjustment in chinese children: A longitudinal study. Journal of Family Psychology, 14(3), 401-419.

Connell, C. M., \& Prinz, R. J. (2002). The impact of childcare and parent-child interactions on school readiness and socia skills development for low-income African American children. Journal of School Psychology, 40(2), 177-193.

Cummings, E. M. (1995). Security, emotionality, and parental depression: A commentary. Developmental Psychology, 31 425-427.

D'Avila-Bacarii, K. M. G., Marturano, E. M, \& Elias, L. C. do S. (2005). Recursos e adversidades no ambiente familiar de crianças com desempenho escolar pobre. Paidéia, 15(30), 43-55.

Dessen, M. A., \& Polonia, A. C. (2007). Família e escola. Paidéia 17(36), 21-32.

Feinberg, M. E., Button, T. M., Neiderhiser, J. M., Reis, D. \& Hetherington, E. M. (2007). Parenting and adolescent antisocial behavior and depression: Evidence of genotype $x$ parenting environment interaction. Archives of General Psychiatry, 64, 457-465.

Ferriolli, S. H. T., Marturano, E. M., \& Puntel, L. P. (2007) Contexto familiar e problemas de Saúde Mental infantil no Programa de Saúde da Família. Revista Saúde Pública 41(2), 251-259.

Goodman, A., Fleitlich-Bilyk, B., Patel, V., \& Goodman, R. (2007). Child, family, school and community risk factors for poor mental health in Brazilian school children. Journal of American Academy of Child and Adolescence Psychiatry, 46, 448-456.

Gomide, P. I. C. (2004). Pais presentes, pais ausentes: regras e limites. Petrópolis, RJ: Vozes.

Hollingshead, A. B. (1975). Four factor index of social status. Department of Sociology, Yale University, unpublished working paper.

Keown, L. J., \& Woodward, L. J. (2002). Early parent-child relations and family functioning of preschool boys with pervasive hiperactivity. Journal of Abnormal Child Psychology, $30(6), 541-553$

Kreppner, K. (2000). The child and the family: Interdependence in developmental pathways. Psicologia: Teoria e Pesquisa, 16(1), 11-22.

LaFreniere, P. J., \& Dumas, J. E. (1996). Social competence and behavior evaluation in children ages 3 to 6 years: The short form (SCBE-30). Psychological Assessment, 8, 369-377.

Mondin, E. M. C. (2005). Interações afetivas na família e na pré-escola. Estudos de Psicologia, 10(1), 131-138, Moura, C. B., Marinho-Casanova, M. L., Meurer, P. H., \& Campana, C. (2008). Caracterização da clientela pré-escolar de uma clínica-escola brasileira a partir do Child Behavior Checklist (CBCL). Contextos Clínicos, 1(1), 1-8.

Pacheco, M. M. B. \& Sisto, F. F. (2005). Ajustamento social e dificuldade de aprendizagem. Psic, 6(1), 43-50.

Patterson, G., Reid, J., \& Dishion, T. (1992). Antisocial boys. Eugene: Castalia.

Pesce, R. (2009). Violência familiar e comportamento agressivo e transgressor na infância: uma revisão da literatura. Ciência \& Saúde Coletiva, 14(2), 507-518.

Pekrun, R. (2001). Familie, Schule und Entwicklung. In S. Walper \& R. Pekrun (Eds.), Familie und Entwicklung. Aktuelle Perspektiven der Familienpsychologie. Göttingen, Germany: Hogrefe.

Prado, A. B. (2005). Semelhanças e diferenças entre homens e mulheres na compreensão do comportamento paterno. Dissertação de mestrado. Programa de Pós-Graduação em Psicologia, Universidade Federal de Santa Catarina, Florianópolis, SC.

Ribas Jr., R. C., Seidl de Moura, M. L., Gomes, A. A. N., Soares, I. D., \& Bornstein, M. H. (2003). Socioeconomic status in Brazilian psychological research. Part 1: Validity, measurement, and application. Estudos de Psicologia, 8, 375-383.

Rocha, M. M., \& Brandão, M. Z. da S. (2001). A importância do auto-conhecimento dos pais na análise e modificação de suas interações com os filhos. In M. Deletti (Org.), Sobre comportamento e cognição (Vol. 2, pp. 133-141). Santo André: ESETEc Editores Associados.

Rollett, B., \& Werneck, H. (2002). Klinische Entwicklungspsychologie der Familie. Aufgaben und Perspektiven. In B. Rollet \& H. Werneck (Eds.), Klinische Entwicklungspsychologie der Familie. (pp. 245-267). Göttingen, Germany: Hogrefe.

Szelbracikowski, A. C., \& Dessen, M. A. (2007, jan./abr.). Comportamento exteriorizado e relações familiares. Psicologia em Estudo, 12(1), 33-40.

Teodoro, M. L. M., \& Käppler, K. C. (2003). Familiograma: desenvolvimento de um novo instrumento para a avaliação das relações familiares (Psicopatologia do Desenvolvimento Relatórios Técnicos, pp. 2-21). Universidade Federal de Minas Gerais, Laboratório de Neuropsicologia do Desenvolvimento e Laboratório de Psicologia da Família, Belo Horizonte, MG.

Teodoro, M. L. M. (2006). Afetividade e conflito em díades familiares: avaliação com o Familiograma. Revista Interamericana de Psicologia, 40(3), 385-390.

Teodoro, M. L. M., Cardoso, B. M., \& Freitas, A. C. H. (No prelo), Afetividade e conflito familiar e sua relação com a depressão em crianças e adolescentes.

Zamberlan, M. A. T. (2002). Interação mãe-criança: enfoques teóricos e implicações decorrentes de estudos empíricos. Estudos de Psicologia, 7(2), 399-406. 